\title{
An Inspection Method of Rice Milling Degree Based on Machine Vision and Gray-Gradient Co-occurrence Matrix
}

\author{
Peng $\mathrm{Wan}^{1,2}$ and Changjiang Long ${ }^{1, *}$ \\ ${ }^{1}$ College of Engineering, Huazhong Agricultural University, Wuhan, P.R. China \\ ${ }^{2}$ College of Biological and Agricultural Engineering, Jilin University, Changchun, P.R. China \\ wanpeng09@mail.hzau.edu.cn, longchj@mail.hzau.edu.cn
}

\begin{abstract}
A detection method of the rice milling degree was proposed based on machine vision with gray-gradient co-occurrence matrix. Using an experimental mill machine, different milling degree samples of rice were prepared. The rice kernel image of the different milling degree was get by a machine vision detecting system, then the texture features of the rice image were obtained by using gray-gradient co-occurrence matrix, at last the Fisher discriminate functions constructed using stepwise discriminate analysis were used to detect the milling degree of the rice samples. The testing results show that the average accuracy rate of the different milling degree detected using the method of 4 rice samples is $94.00 \%$.
\end{abstract}

Keywords: Milling degree, Machine vision, Gray-gradient co-occurrence matrix, Fisher discriminance, Rice.

\section{Introduction}

The rice process precision is the embryo extent of the processed rice, for the other saying is how much the embryo of the brown rice have been removed, referring to brown rice is milled to the extent of the cortex, at the same time that is the appearance quality of rice and the main indicator advantages and disadvantages of the performance evaluation of rice quality. According to the rules of "GB 1354-2009 rice," the process precision of rice by machining accuracy can be divided into one, two, three and four levels. There are several means to evaluate the processing, the national standard to provide for the direct comparison method or staining for detection, were evaluated through artificial sense of concept, but these methods are affected by light conditions, subjective feelings or many other factors and the accuracy is not high. The complicated operation process, low detection efficiency, and low accuracy can not meet the rapid, objective, accurate detection needs[1].

Detected by image analysis of rice processing is a very popular research direction [2-4]. Different levels of precision processing make the surface texture of the rice

\footnotetext{
* Corresponding author.
} 
different. This paper uses machine vision technology to study the surface texture analysis and explore the relationship between precision of rice correspondence and characteristics of surface texture, in order to achieve accuracy by processing rice through testing the surface texture of rice.

There are several means of image texture analysis[5-6], this paper use graygradient co-occurrence matrix to analysis the surface texture characteristics of rice.

\section{Gray-Gradient Co-occurrence Matrix Detection Method}

Gray-gradient co-occurrence matrix embodies the relationship between gray and gradient of each pixel. Gray of each pixel constitute the basis of an image, and gradient constitute the elements edges of the image which provide the main image information[7]. Therefore, the gray-gradient co-occurrence matrix considers the Joint Statistical Distribution of the gray and the size of the edge gradient of each pixel.

The operational processes of how to extract the gray-gradient co-occurrence matrix from the image is shown in Figure 1.

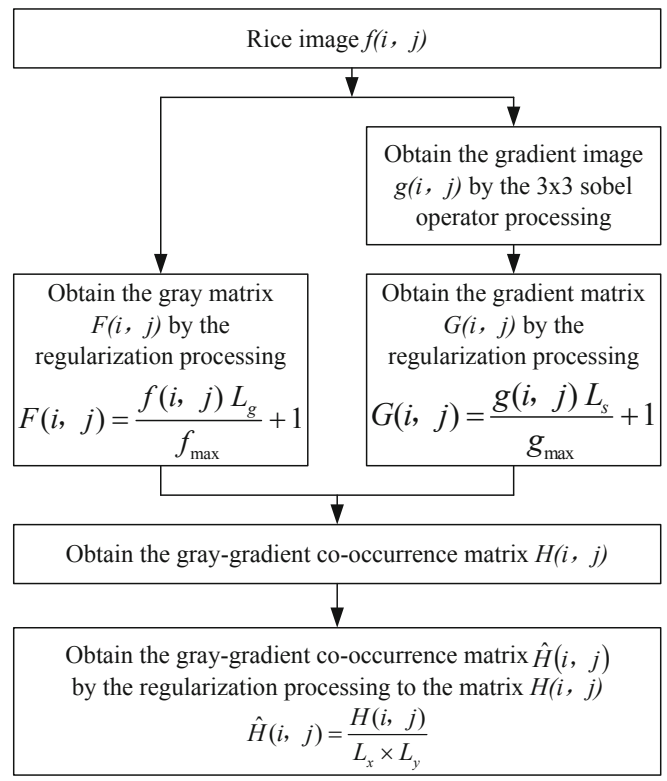

Fig. 1. The operational processes of how to extract the gray-gradient co-occurrence matrix from the image

Gray-gradient co-occurrence matrix describes the distribution for the gray and gradient of each pixel in an image and the spatial relations between pixels. It is the complex of the gray information and the gradient information, for the reason that we can extract the characteristic parameters of each image from the gray-gradient co-occurrence matrix. 
This paper differently collects 15 texture index from the images of the rice grains, such as small gradient strengths(R1), large gradient strengths (R2), gray uneven representation(R3), gradient uneven representation(R4), energy(R5), gray mean(R6), gradient mean(R7), gray mean square(R8), gradient mean square(R9), relevance(R10), gray entropy(R11), gradient entropy(R12), mixing entropy(R13), inertia(R14), inverse gap(R15).

\section{The Detection of Rice Processing Accuracy}

\subsection{Preparation of the Samples}

We selected the Wan Chang Rice which was produced in Jilin Province as the samples for the rules research of detection accuracy of the rice. Three rice samples were weight, each $200 \mathrm{~g}$. First, the experimental husker was used to shell the paddy into brown rice; then, the brown rice was milled by the milling machine, and collected the rice samples with different precision. As the milling process, the longer you milled, the more brown rice to be grinded, and the different precision you could get. We set three different milling time as 30s, 60s, 90s to milling the paddy, and get three different brown rice as S1, S2, S3. At the same time, we put the Supermarket Wan Chang rice as $\mathrm{S} 4$ for the detection accuracy for rice processing test.

\subsection{The Acquisition of Rice Image}

In order to obtain rice samples images, we designed a vision inspection system for rice precision.

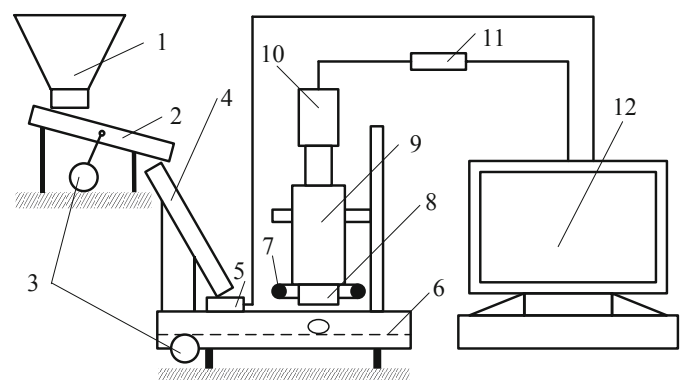

Fig. 2. Rice precision machine vision inspection system. 1.hopper 2.V-groove 3.electric motors 4.drop tank 5.count sensor 6.conveyor 7.Ring light source 8.shot 9.stereomicroscope 10.ccd camera 11.image acquisition card 12.computer software.

When detecting, put the rice samples into the hopper, then the V-groove vibrated after the motor driven and made the rice samples fall into the $\mathrm{V}$-groove and ranked to move forward, when the rice grain in V-groove fallen into the drop tank it would accelerate the decline. The transmission worked when the count sensor fallen on the 
conveyor belt, and the count sensor put the information into the computer; When the rice grains through the camera lens, the corn grain shape detection software collected all the images and sent these images to the computer for detection.

This paper used a microscope to MOTIC stereomicroscope; camera produced in Japan SONY DXC-390P 3 CCD Color camera; image acquisition card produced by the Canadian CronosPlus image acquisition card; conveyor belt for the light blue canvas belt; circular fluorescent light tubes with $5 \mathrm{~W}$; major rice-shaped detection software written using Visual $\mathrm{C}++6.0$.

\subsection{Rice Images Process}

The operation process of the obtained rice images was shown in Figure 3.

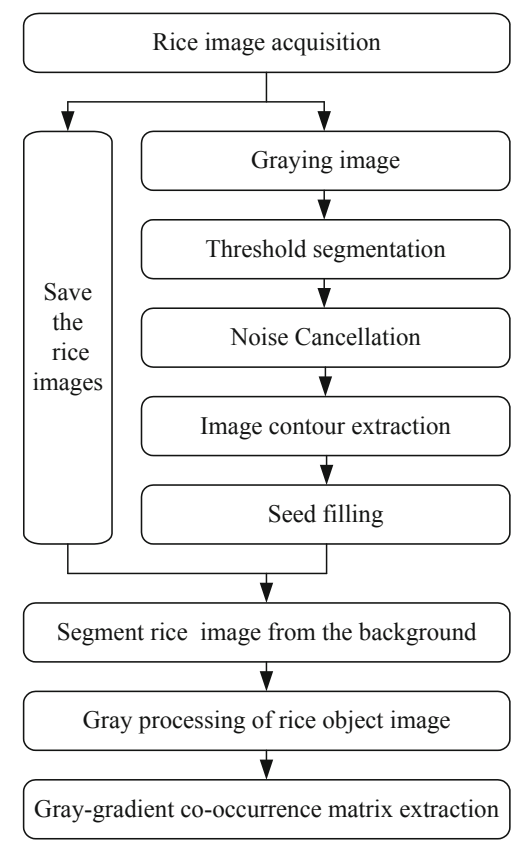

Fig. 3. Rice image preprocess procedure

\section{Results and Discussion}

\subsection{The Extraction for the Rice Samples of Different Precision Texture Feature}

From the 4 different rice samples, each sample was selected 300 full grain rice, to get 1200 different images, and preprocess those images to get the original image of rice with different precision. 


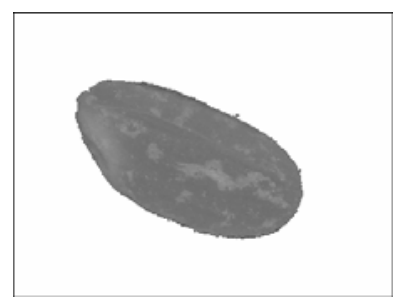

(1)

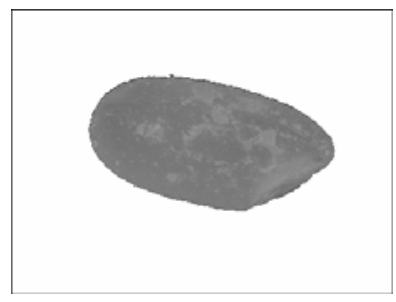

(3)

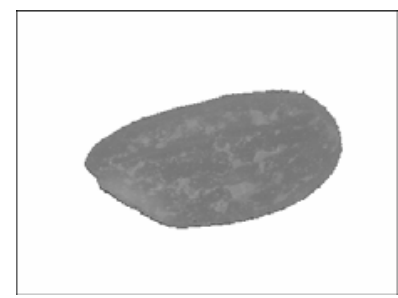

(2)

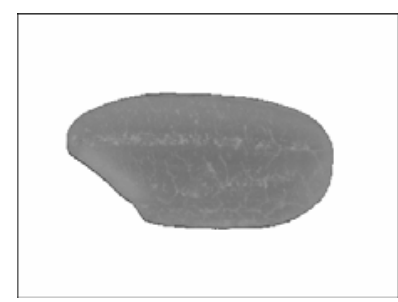

(4)

Fig. 4. The original image of rice samples of different precision. (1) Rice sample S1; (2) Rice sample S2; (3) Rice sample S3; (4) Rice sample S4.

From the images, the rice of different precision, its surface texture patterns significantly different. There-fore, we could use the gray-gradient co-occurrence matrix to test the precision of rice. Preprocess to the original image of the rice samples, we get gray-gradient co-occurrence matrix of the rice image, then use the image to collect the texture data, each sample get 300 sets of data.

Table 1. The parameter mean of the rice samples texture

\begin{tabular}{ccccc}
\hline \multirow{2}{*}{ Texture parameters } & \multicolumn{4}{c}{ Number of rice samples } \\
\cline { 2 - 5 } R1 & $\mathrm{S} 1$ & $\mathrm{~S} 2$ & $\mathrm{~S} 3$ & $\mathrm{~S} 4$ \\
\hline R2 & 0.2398 & 0.2407 & 0.2411 & 0.2346 \\
R3 & 6.4022 & 6.1596 & 6.0700 & 6.0915 \\
R4 & 0.3990 & 0.3947 & 0.3876 & 0.3509 \\
R5 & 0.8339 & 0.8434 & 0.8484 & 0.7790 \\
R6 & 0.3365 & 0.3371 & 0.3323 & 0.2813 \\
R7 & 10.6028 & 11.0306 & 11.0789 & 11.1660 \\
R8 & 1.6924 & 1.6712 & 1.6668 & 1.7152 \\
R9 & 4.1985 & 4.2574 & 4.1795 & 3.9872 \\
R10 & 2.2332 & 2.1884 & 2.1677 & 2.1366 \\
R11 & 0.0582 & 0.0406 & 0.0474 & 0.0369 \\
R12 & 1.3577 & 1.3289 & 1.3317 & 1.4491 \\
R13 & 0.5907 & 0.5714 & 0.5643 & 0.6856 \\
R14 & 1.8796 & 1.8288 & 1.8238 & 2.0411 \\
R15 & 103.5886 & 112.5006 & 112.7364 & 112.1271 \\
Category values & 0.0219 & 0.0210 & 0.0210 & 0.0207 \\
\hline
\end{tabular}


200 sets of data were selected from each texture feature data of rice samples as the test samples, and the other 100 sets data as the testing samples. 1,2,3,4 were used as the numerical classification number of rice samples of S1, S2, S3, and S4.

\subsection{Detection by Stepwise Discriminant Analysis of Rice Processing Accuracy}

Discriminant analysis[8] is based on the known samples to build a discriminant function group, To make the Makes the minimum rate of wrongful convictions in the identification of the sample classification. Analysis variables by the contribution of independent variable, and Introduction and removing variables one by one, until no new significant effects of independent variables could be neither introduced, nor no significant effect of the independent variables are removed from the equation so far.

Adopt the discriminant analysis of group $\mathrm{F}$ by the smallest value than the maximum choice of variables. Select when the F value is greater than 7.68 will join the variable into the discriminant function and when the $\mathrm{F}$ value is less than 1.355 will remove the variable from the discriminant function as the criterion, Using SPSS software testing 800 samples to analyze the data set of texture features, Calculation the Fisher discri-minant function which can be used for identification and classification for unknown samples, Fisher discriminant function is available as Table 2 shows the coefficient.

Table 2. The coefficients of the Fisher discriminant function

\begin{tabular}{ccccc}
\hline \multirow{2}{*}{ Texture parameters } & \multicolumn{4}{c}{ Classification } \\
\cline { 2 - 5 } & $\mathrm{S} 1$ & $\mathrm{~S} 2$ & $\mathrm{~S} 3$ & $\mathrm{~S} 4$ \\
\hline R1 & 26456.25 & 26448.55 & 26467.79 & 26506.75 \\
R3 & 526.18 & 517.53 & 514.82 & 519.44 \\
R5 & -425.70 & -420.82 & -420.89 & -423.64 \\
R6 & 11.07 & 11.20 & 11.32 & 11.37 \\
R7 & 1173.44 & 1174.01 & 1176.92 & 1179.87 \\
R8 & 3.27 & 2.95 & 2.67 & 2.63 \\
R9 & -269.99 & -270.05 & -270.69 & -271.51 \\
R11 & 520.78 & 517.03 & 517.03 & 522.35 \\
R12 & 1260.50 & 1259.41 & 1259.93 & 1264.28 \\
R13 & -425.09 & -423.56 & -424.55 & -428.64 \\
constant & -3965.38 & -3960.24 & -3965.50 & -3980.36 \\
\hline
\end{tabular}

We can see from table 3, F satisfy the stepwise discriminant analysis into the discriminant function value greater than 7.68 and $F$ value is less than 1.355 , when the variables for the discriminant function to remove a small gradient strengths(R1), representation of uneven gray(R3), energy(R5), mean of gray(R6), mean of gradient(R7), MES of gray(R8), MES of gradient(R10), gray entropy(R11), gradient entropy (R12), gray entropy(R13) 10 texture parameters, etc.. Other variables did not meet the criteria for variable tick was removed from discriminant function. 
According to Table 3, construction of Fisher discriminant function groups were as follows:

$$
\begin{aligned}
Q_{1}= & 26456.25 R_{1}+526.18 R_{3}-425.70 R_{5}+11.07 R_{6} \\
& +1173.44 R_{7}+3.27 R_{8}-269.99 R_{9}+520.78 R_{11} \\
& +1260.50 R_{12}-425.09 R_{13}-3965.38 \\
Q_{2}= & 26448.55 R_{1}+517.53 R_{3}-420.82 R_{5}+11.20 R_{6} \\
& +1174.01 R_{7}+2.95 R_{8}-270.05 R_{9}+517.03 R_{11} \\
& +1259.41 R_{12}-423.56 R_{13}-3960.24 \\
Q_{3}= & 26467.79 R_{1}+514.82 R_{3}-420.89 R_{5}+11.32 R_{6} \\
& +1176.92 R_{7}+2.67 R_{8}-270.69 R_{9}+517.03 R_{11} \\
& +1259.93 R_{12}-424.55 R_{13}-3965.50 \\
Q_{4}= & 26506.75 R_{1}+519.44 R_{3}-423.64 R_{5}+11.37 R_{6} \\
+ & 1179.87 R_{7}+2.63 R_{8}-271.51 R_{9}+522.35 R_{11} \\
+ & 1264.28 R_{12}-428.64 R_{13}-3980.36
\end{aligned}
$$

\begin{tabular}{|c|c|c|c|c|c|}
\hline \multirow{2}{*}{ samples } & \multicolumn{4}{|c|}{ the forecast of rice sample precision } & \multirow{2}{*}{ total } \\
\hline & S1 & S2 & S3 & S4 & \\
\hline S1 & 93 & 7 & 0 & 0 & 100 \\
\hline S2 & 3 & $\underline{95}$ & 2 & 0 & 100 \\
\hline S3 & 0 & 5 & $\underline{92}$ & 3 & 100 \\
\hline S4 & 0 & 0 & 4 & $\underline{96}$ & 100 \\
\hline correct rate $/ \%$ & 93.00 & 95.00 & 92.00 & 96.00 & \\
\hline average correct rate $/ \%$ & \multicolumn{4}{|c|}{$94.00 \%$} & \\
\hline
\end{tabular}

Formula(1), (2), (3), (4) form fisher discriminant function group, the discriminant function groups were used in the category test for the precision of the unknown rice samples, put the data of the rice texture characteristics into the 4 discriminant function, and get the 4 discriminant function value, which one got the largest discriminant function value it is the most precision one.

According to 4 different rice precision test sample images, use gray-gradient cooccurrence matrix to collect texture parameters of rice and use Fisher discriminant function to test classification accuracy of the precision of rice sample, accuracy as shown in Table 3.

Table 3. Classification of the test samples of rice processing precision

Prompt: The data underlined detect the correct samples of rice grains.

From the table 3, using Fisher discriminant function to test the 4 different precision of rice samples, for class $\mathrm{S} 1$, the accuracy of detection for rice samples was $93.00 \%$, and $95.00 \%$ for class S2, 92.00\% for class S3, the class S4 get the highest accuracy of $96.00 \%$. The average accuracy rate of the 4 different precision process is $94.00 \%$. 


\section{Conclusions}

This paper designed a vision inspection system for rice precision testing to get the image of rice sample, and the texture feature parameters of the different precision rice samples were collected by using gray-gradient co-occurrence matrix, and then constructed Fisher discriminant groups to detect the rice precision. The results show that 400 rice samples of 4 different process precision can reach the average correct to $94.00 \%$; The accuracy of rice samples for processing 30S (the S1 class) was $93.00 \%$, for processing 60S (the S2 class) was $95.00 \%$, for processing $90 \mathrm{~S}$ (the S3 class) was $92.00 \%$, the milled rice (the S4 class) could get the highest rate of $96.00 \%$. So, this method can be used to detect the rice process precision.

\section{References}

1. Huang, X., Fang, R., Wu, S.: Progress in research of detection method for degree of rice milling. Journal of JiangSu University of science and technology 9(3), 6-9 (1998)

2. Tian, Q.: Application of the computer image processing technique in discerning the degree of rice whiteness. Cereal and Feed Industry (10), 10-11 (1997)

3. $\mathrm{Xu}, \mathrm{L} .$, Qian, M., Fang, R.: Image process technique to cognize the external qualities and mil-ling degree of rice. Transactions of the chinese society of agricultural Engineering 12(3), 176-179 (1996)

4. Zhang, H., Meng, Y., Zhou, Z.: Compounds, quantitative analyzing rice milling degree based on digital image technology. Journal of the Chinese Cereals and Oils Association 21(4), 187-190 (2006)

5. Li, B.: Study of image texture analysis and classification method. Fudan University, Shanghai (2007)

6. Sheng, W., Liu, J.: Image texture analysis methods and recent advances. Radio Engineering 28(5), 8-13 (1998)

7. Hong, J.: Gray level-gradient co-occurrence matrix texture snalysis method. Acta Automatica Sinica 10(1), 22-25 (1984)

8. Zhong, C., Guo, Q.: Fisher discrimination method and its application. Journal of Southwest Jiaotong University 43(1), 136-141 (2008) 